\title{
Intersektionalität privilegierter Identitätsdimensionen
}

\author{
Performanz einer weißen, männlichen und globalen Elite
}

\author{
Lars Meier
}

Privilegierte männliche, hochmobile Finanzmanager sind nicht nur Teil abstrakter ortsübergreifender Netzwerke, sondern sie arbeiten an konkreten Arbeitsorten. Diese Arbeitsorte werden von ihnen auch in Abhängigkeit zur sozialen Identität wahrgenommen und betrachtet. Am Beispiel von deutschen Finanzmanagern in London und Singapur wird die Relevanz des lokalen Arbeitsortes für die Identitätsaufführungen der globalen Elite mit einer intersektionalen Perspektive herausgearbeitet. Da die Performanz dieser Identitätsdimension als dynamischer Prozess verstanden wird - also als eine Dynamik, in der diese im Handeln alltäglich erzeugt und aufgeführt wird - werden die alltäglichen Aufführungen der Globalen Elite in ihrer Beziehung zu dem Arbeitsort, an dem die Aufführungen stattfinden, analysiert. Es wird deutlich, dass mit dem Ansatz der Intersektionalität auch privilegierte Identitätsdimensionen in den Blick genommen werden können. Es zeigt sich, dass sich in Abhängigkeit vom Ort der Performanz die Identitätsdimension ,globale Elite' differenziert und sich in spezifischer Weise mit der Identitätsdimension des Weißseins überschneidet.

Ersteinreichung: 5. Februar 2018; Veröffentlichung online: 28. November 2018

An english abstract can be found at the end of the document.

\section{Einführung}

Die internationale Finanzwelt ist als Heils- und Krisenbringer in einem ständigen Licht der Aufmerksamkeit. Mit ihren computergestützten, in Echtzeit ablaufenden internationalen Austauschprozessen von Informationen und Finanzkapital gelten die Aktivitäten dieser Branche im besonderen Maße als Paradebeispiele von grenzüberschreitenden Globalisierungsprozessen. Die in dieser Branche arbeitenden Manager, die für ihre Arbeit in ein anderes Finanzzentrum ziehen und von dort aus über die Nutzung von Kommunikations- und Transportmitteln immer wieder in Kontakt zu anderen internationalen Finanzzentren stehen, erscheinen dabei meistens als vom Ort ihrer Handlung entkoppelte hochmobile transnationale Netzwerkbildner. Sie werden als Angehörige einer "global hightechnology professional culture“ (Knorr Cetina/Bruegger 2002) verstanden, die sich vom Ort gelöst hat und in der Zeit lebt (Castells 2000). Finanzmanager - in diesem Fall ist die maskuline Form bewusst gewählt - finden sich auch in populären medialen Darstellungen, wie der des rücksichtslos nach Erfolg und Gewinn strebenden Gordon Gecko im Film Wall Street von Oliver Stone, des Brokers Sherman McCoy, der sich in dem Roman Fegefeuer der Eitelkeiten von Tom 
Wolfe (1987) als ,Master of the Universe“ fühlt oder des Vermögensverwalters Eric Parker im Roman Cosmopolis von Don DeLillo, der in seiner StretchLimousine auf der Fahrt durch Manhattan auch Armut begegnet. Die privilegierte Position der Finanzmanager und die soziale Ungleichheit in der Begegnung mit ,Anderen', den weniger privilegierten und ihren städtischen Räumen, wird zuweilen in medialen Texten sichtbar, ist aber wissenschaftlich nur wenig erforscht.

Soziolog_innen wie Manuel Castells oder Saskia Sassen diagnostizieren eine soziale Zweiteilung zwischen den mobilen Hochqualifizierten wie den Finanzmanagern und den an den Ort gebundenen Unterprivilegierten, oder wie es Zygmunt Baumann ausdrückt: „Some inhabit the globe; others are chained to place“ (Bauman 1998: 45). Auf Basis einer solchen Perspektive war die Forschung zu den mobilen Finanzmanagern und Professionals lange davon dominiert, diese in erster Linie durch ihre hohe Mobilität und Einbindung in transnationale Netzwerke zu betrachten. Mit ihren Reisen, transnationalen Geschäftskontakten und häufigen Wohnortwechseln galten sie lange Zeit als Musterbeispiele einer „borderless world“ (Ohmae 1990). Dabei wurden ihre alltäglichen Praktiken häufig auf ihre Kommunikationsund Mobilitätspraktiken und die transnationale Netzwerkbildung (Caroll/ Fennema 2002, Salt 1997, Van der Pijl 1998) zwischen den Global Cities (Sassen 2001) reduziert. Das hatte zwei Konsequenzen:

Erstens wird der städtische Ort in dieser Perspektive zu einem für die Handlung der Finanzmanager unbedeutenden Rahmen, er wird zu einem als einheitlich konzipierten globalen Ort. Diese Reduktion der Alltagspraktiken einer als ortlos betrachteten „transnational class“ (Sklair 2001) auf ihre grenzübergreifenden globalen Praktiken, geht mit einer Vernachlässigung von lokalen handlungsrelevanten Spezifika einher (Ley 2004, Willis/Yeoh/ Fakhri 2002, Fechter 2007).

Daraus ergibt sich zweitens, dass alltägliche soziale Ungleichheitsprozesse, die vor Ort relevant werden, aus dem Blick geraten sind. Daher gab es auch lange Zeit einen Mangel an Untersuchungen zur sozialen Ungleichheit, die privilegierte Gruppen, wie die Finanzmanager, in ihrem Alltagshandeln aus einer Perspektive sozialer Ungleichheit in den Blick genommen haben (Korsnes et al. 2017, Meier 2015a). Die klassischen sozialen Ungleichheitsdimensionen Klasse, Geschlecht und ,Ethnizität‘ wurden damit in ihrer Relevanz für das Alltagshandeln und die soziale Identitätsbildung der privilegierten Gruppen kaum berücksichtigt.

Mein Aufsatz untersucht die Relevanz des städtischen Ortes für alltägliche Ungleichheitsprozesse der privilegierten mobilen Finanzmanager. Mit dem vorgenommenen Vergleich der Performanz der deutschen Finanzmanager in London und Singapur werden im besonderen Maße die Spezifika beider Städte in ihrer Wirkung auf die Performanz privilegierter Identitäten deutlich.

Nach einer Darstellung der angewendeten qualitativen Methoden (Interviews und Beobachtungen) und der Datengrundlage im dritten Abschnitt verdeutlicht der vierte Abschnitt zunächst die Qualitäten, die es erlauben, von einer sozialen Identitätsdimension der globalen Elite auszugehen. Vor diesem Hintergrund analysiere ich im fünften Abschnitt die Performanz als globale Elite in London und im sechsten Abschnitt die in Singapur. 
Ausgehend von der Identitätsdimension globale Elite werden im abschließenden siebten Abschnitt besonders die Intersektionalität von Weißsein mit Klasse (globale Elite) und Geschlecht herausgearbeitet und die ortsabhängigen Differenzierungen der Performanz gezeigt.

Wie sich noch zeigen wird, variiert die genaue Ausprägung der Identitätsdimensionen innerhalb einer gewissen Bandbreite in Abhängigkeit vom Ort ihrer Performanz. Zunächst gilt es aber im zweiten Abschnitt das Konzept der Intersektionalität vorzustellen und die Relevanz des Ortes in dieser Perspektive auf soziale Ungleichheiten herauszuarbeiten.

\section{Intersektionalität und die Performanz von Identitäten am Ort}

Der Begriff Finanzmanager, die mediale Darstellung der Finanzmanager und die entsprechenden Beschäftigungsquoten veranschaulichen, dass die Figur des Finanzmanagers stark männlich konnotiert ist. Auch dieser Aufsatz nimmt männliche Finanzmanager in den Blick, allerdings ausdrücklich vor dem Hintergrund Männlichkeit als Dimension sozialer Ungleichheit zu verstehen. Ebenso wie Männlichkeit wird auch das Weißsein häufig nicht im Kontext sozialer Ungleichheit beachtet und bleibt als privilegiertes und normgebendes Strukturelement unsichtbar. Weißsein bezieht sich dabei nicht auf eine Hautfarbe, auf deren Basis häufig vorgeblich homogene Gruppen mit entsprechenden Zuschreibungen konstruiert werden. Vielmehr bezeichnet Weißsein eine Position im Gefüge der sozialen Ungleichheit - nämlich die privilegierte Position - die immer in Beziehung zu den durch Rassismus diskriminierten Schwarzen steht (auch dies bezeichnet keine Hautfarbe, sondern die unter Rassismus leidende Gruppe) (Frankenberg 1993, Kerner 2013). Um die soziale Position der Finanzmanager im Gefüge sozialer Ungleichheit zu begreifen, ist es also notwendig die Multidimensionalität von Identität und sozialen Platzanweisern zu berücksichtigen und den Fokus nicht nur auf Klasse (globale Elite), sondern auch auf Geschlecht (Männlichkeit) und ,Ethnizität‘ (Weißsein) zu richten.

Der Ansatz der Intersektionalität hat eine solche Verschränkung von Kategorien sozialer Ungleichheit in den Blick genommen (Lutz/Herrera Vivar/Supik 2010, Winker/Degele 2009). Entwickelt wurde dieser Ansatz vor dem Hintergrund der postkolonialen Kritik von schwarzen Feministinnen (Crenshaw 1989, Collins 1998, hooks 1981) daran, dass der Feminismus zwar die Unterdrückung von weißen Frauen der Mittelschicht thematisiert, aber nicht die besondere Situation von schwarzen Frauen betrachtet hat. Es sind jedoch Differenzierungen notwendig; oder anders gesprochen: Frauen sind auch sozialstrukturell betrachtet nicht gleich. Dies zeigt sich an dem Beispiel von schwarzen Haushaltshilfen, deren Arbeit es ihren weißen Arbeitgeberinnen ermöglicht einer besser entlohnten und angeseheneren Lohnarbeit nachzugehen. Der Ansatz der Intersektionalität berücksichtigt diese Differenz zwischen den Frauen, indem nunmehr verschiedene Diskriminierungsformen (besonders Rassismus und Sexismus) in ihrer gegenseitigen Verschränkung und Wechselwirkung betrachtet werden.

Am Intersektionalitätsansatz wird kritisiert, dass dabei undefiniert bleibt welche Kategorien sozialer Ungleichheit (wie Gender, Gesundheit, ,Ethnizität‘, Klasse, Sexualität, Körper, Gesundheit, Alter) berücksichtigt werden müssen. 
Dies kann aber auch gerade als besondere Stärke des Ansatzes gesehen werden, denn so ist es möglich auch „fortwährend für neue mögliche Auslassungen, Entnennungen und Exklusionen sensibel zu bleiben“ (Lutz/Herrera Vivar/ Supik 2010: 12). Der Intersektionalitätsansatz geht von Kategorien sozialer Ungleichheit aus, die mit der Benennung auch reproduziert werden, was verschiedentlich kritisiert wurde (Budde 2013). Es ist ohne Zweifel grundsätzlich hochproblematisch, wenn Kategorien sozialer Ungleichheit mit stereotypischen Zuschreibungen von Eigenschaften und der Bildung von vorgeblich homogenen Gruppen verbunden werden. Zum Erkennen der Strukturen sozialer Ungleichheiten und auch zu ihrer Benennung sind Bezüge zu solchen Kategorien jedoch notwendig. Wie sollten beispielsweise Sexismus, Klassismus oder Rassismus in den Blick genommen werden, wenn nicht die darunter leidenden (oder die davon profitierenden) Gruppen benannt werden?

Der BegriffIntersektionalität impliziert durch die Metapher der Straßenkreuzung (deutsch für intersection), dass vor und nach der Kreuzung die Kategorien sozialer Ungleichheit unabhängig und isoliert voneinander bestehen. Um die Dauerhaftigkeit der Überlagerungen der Ungleichheitskategorien treffender zu kennzeichnen, wurde daher vorgeschlagen den Begriff der Interdependenzen vorzuziehen (Walgenbach 2012). Da es sich bei Intersektionalität um ein mittlerweile etabliertes internationales Konzept handelt, wird dieser in meinem Aufsatz einer neuen Begriffsinnovation vorgezogen.

Vor dem Hintergrund der Wurzeln des Intersektionalitätsansatzes ist es wenig überraschend, dass Forschung, die sich auf diesen Ansatz bezieht, auf Diskriminierungen fokussiert und ihn nicht anwendet, um Verschränkung der Kategorien sozialer Privilegierungen in den Blick zu nehmen. Ein Problem ist daher: „the curious tendency to neglect analysis of the power of the actions of the dominant group within the 'category'. The analysis of intersectionality has often focused on the actions of the disadvantaged groups. For example, there has been a focus on the actions of white women rather than white men [...]“ (Walby/Armstrong/Strid 2012: 230). Mein Aufsatz will mit der Analyse der Intersektion der privilegierten Kategorie globale Elite (Klasse) mit der des Weißseins (,Ethnizität') und der von Männlichkeit (Geschlecht) dazu beitragen diese Forschungslücke zu schließen.

Dabei ermöglicht es der Intersektionalitätsansatz auch auf der Alltagsebene der Handlungen, der sprachlichen Aussagen und symbolischen Markierungen von ,Fremd“ und ,fremden Räumen“ machtvolle Privilegierungen als „lived experiences“ (Valentine 2007) in den Blick zu bekommen. Der Ansatz der Performanz von Judith Butler (1991) bietet sich an, um diese Verbindung von alltäglichen Handlungen und Aussagen zu machtvollen Privilegierungen sozialer Identitäten (wie Weißsein, Männlichkeit und globale Elite sein) zu analysieren. Denn: Kategorien sozialer Ungleichheit sind nicht nur analytische Kategorien, sondern auch Dimensionen sozialer Identitäten, die eine gewisse Dynamik aufweisen. Sie sind „neither fixed nor singular; rather it is a constantly changing relational multiplicity" (Brah 1996: 123) und werden in alltäglichen Handlungen und Sprechweisen (re-)produziert (Butler 1991, Meier 2009). Für ihre alltägliche Re-produktion sind die Konstruktion von ,Eigen“ und ,Fremd“ (Bhabha 1994, Hall 1996) besonders bedeutsam. Die Verschränkungen der Identitätsdimensionen, die Kategorien sozialer Ungleichheit darstellen, werden in diesem Aufsatz in Abhängigkeit vom Ort 
ihrer alltäglichen Performanz und Verkündung betrachtet. Butler hat das Konzept der „Performanz der Geschlechtsidentitäten (gender performance)“ (Butler 1991: 202, Hervorhebung im Original) eingeführt, um zu betonen, dass Geschlecht dargestellt wird. Damit entnaturalisiert sie Geschlecht und Geschlechtsidentität. Die „konstruierten Akte, Gesten und Inszenierungen erweisen sich insofern als performativ, als das Wesen oder die Identität, die sie angeblich zum Ausdruck bringen, vielmehr durch leibliche Zeichen und andere diskursive Mittel hergestellte und aufrechterhaltene Fabrikationen/ Erfindungen sind“ (Butler 1991: 200, Hervorhebung im Original). Es gibt vielfältige Studien die eine solche Performanz von Geschlecht belegen. Spezifisch zum Forschungsfeld der vorliegenden Studie analysiert Linda McDowell die Performanz von Männlichkeit und Weiblichkeit bei Finanzbeschäftigten in der Londoner City (McDowell 1997). Auch Weißsein ist eine durch diskursive Mittel hergestellte und aufrechterhaltene Fabrikation, die als sozialer Platzanweiser fungiert und die ich anhand der Aussagen und Handlungen der Finanzmanager an den Orten ihrer Verkündung analysiere.

Mit einem Verständnis, dass Orte „combinations of the material and mental“ (Cresswell 1996: 13) sind, rücken sowohl symbolische Markierungen von Orten (Wacquant 2017) und alltägliche Praktiken an diesen Orten in den Blick des Aufsatzes. Die symbolische Reproduktion von Machtverhältnissen und Ungleichheiten (symbolische Gewalt nach Bourdieu 2005) erfolgt im Zuge der Performanz der privilegierten Identitäten der Finanzmanager. Ein Element dieser Performanz sind spezifische sprachliche symbolische Markierungen von ,anderen“ und ,anderen“ Orten, die als „Ortseffekte“ (Bourdieu 1997) wirksam werden. Ein Ort kann so beispielsweise symbolisch als gefährlich, als kulturell oder als primitiv markiert werden (Jackson 1989, Çınar/Bender 2007). Auch die Begegnung mit, anderen“ ist mitgeprägt durch die symbolische Zuschreibung von bestimmten Eigenschaften, die mit der Identifikation von bestimmten Identitätsdimensionen, wie beispielsweise einem Geschlecht, der ,Ethnizität‘ oder der Milieuzugehörigkeit erfolgt.

Die symbolische Markierung von Orten wird auch auf der Ebene der alltäglichen körperlichen Performanz von Identitäten (durch an die symbolische Markierung angepasste Handlungen oder Emotionen) reproduziert. Bisher wurde dieintersektionale Perspektive auf die Erforschung sozialer Ungleichheit in der Stadtforschung relativ selten angewendet (Valentine 2008). Beispiele sind eine kombinierte Studie aus quantitativer Analyse und Medienkunst zur ungleichen Nutzung des städtischen Raums (Scambor/Zimmer 2012), von Gentrifizierungsprozessen (Frank 2010) oder des ungleichen Risikos städtischer Nachbarschaften (Differenz der sozialen Zusammensetzung) von Umweltschäden betroffen zu sein (Sicotte 2014). Eine Anwendung des Intersektionalitätsansatzes auf die Performanz verschiedener privilegierter Identitäten in ihrer Beziehung zu verschiedenen Städten steht jedoch noch aus.

\section{Soziale Identitäten am Ort analysieren - Methode und Fallauswahl}

Mein Aufsatz basiert teilweise auf überarbeiteten oder in einen neuen $\mathrm{Zu}$ sammenhang gestellten Teilen meiner bereits veröffentlichten Studie „Das Einpassen in den Ort“ (Meier 2009) und enthält damit auch bereits 
veröffentlichte Daten und Textabschnitte. Im Unterschied zu meinen vorherigen Veröffentlichungen (zum Beispiel Meier 2007; Meier 2016, Meier 2015b) wendet der vorliegende Aufsatz das Konzept der Intersektionalität an. Für die Studiehabeich mitLondon undSingapurzweiStädtegewählt, dieim besonderen Maße als international bedeutsame Finanz- und Dienstleistungszentren gelten und die als vernetzte ,global cities' oder ,alpha world cities' (Friedmann 1995, Alderson et al. 2010, Taylor 1994) benannt und in den Konstruktionen globaler städtischer Hierarchien in Toppositionen eingeordnet werden.

Die Studie gründet sich auf 40 in London und Singapur durchgeführte leitfadengestützte, aber in den Nachfragen flexibel gehaltene, episodische Interviews. Interviewt wurden Menschen (vorwiegend Männer) aus unterschiedlichen Alterskategorien mit einem deutschen Pass, von denen sowohl in London als auch in Singapur jeweils 12 in höheren bis höchsten (General Manager, CEO) und 8 in eher mittleren Managementpositionen (Associate Manager) in insgesamt 11 unterschiedlichen Finanzinstitutionen arbeiteten.

Die episodische Interviewform (Flick 2008) ermöglicht es, subjektives Wissen und Alltagserfahrungen zu erheben. Das Interview war gegliedert durch Erzählanregungen und Fragen, die es den Interviewten ermöglichten, erlebte Situationen in London und Singapur zu schildern. Der dafür vorbereitete Interviewleitfaden wurde von mir flexibel, also mit direktem Bezug zu dem von den Interviewpartnern Gesagten, verwendet und der jeweiligen Interviewsituation angepasst. Das Ziel war, es mit Fragen hierzu - also wie es dazu kam, dass sie nach London oder Singapur gekommen sind, zu ihren Lieblingsorten oder mit einer Aufforderung, einen normalen Arbeitstages vom Aufstehen an zu erzählen - den Interviewpartnern zu ermöglichen eigene Erzählungen in der Interviewsituation zu entwickeln. Die meisten Interviews fanden in den Büros der Interviewpartner oder in Kneipen oder Restaurants statt und dauerten 2-4 Stunden. Diese Interviews wurden durch offene ethnografische Interviews und durch Feldbeobachtungen, die sich auf die Handlungen der Finanzmanager und die von ihnen alltäglich genutzten Orte fokussierten, ergänzt. Das ethnographische Interview wurde situativ (bei gemeinsamen Ortsbegehungen) und ohne vorbereiteten Leitfaden entwickelt. Im Unterschied zu einer alltäglichen Unterhaltungssituation stellte ich als Forscher die meisten Fragen und strukturierte damit das Gespräch stärker (Spradley 1979). Die Auswertung der transkribierten Interviews und der Feldnotizen erfolgte nach Prinzipien der Grounded Theory. Dies umfasst eine Offenheit gegenüber Neuem und vorher Unbekanntem in der Erhebung und der Auswertung (anstatt eines hypothesengeleiteten Vorgehens). Außerdem ist damit ein Vorgehen gekennzeichnet, in dem Erhebung und Auswertung ineinandergreifende Phasen sind, wobei die Erhebung (Interviewführung und Beobachtung) an die Zwischenergebnisse der Auswertung angepasst wurde. Die Daten wurden in der Analyse von mir schrittweise kodiert und interpretativ ausgewertet (Charmaz 2014). Die Durchführung von Interviews und Feldbeobachtungen ermöglichte es mir dreierlei zu kombinieren: Die von mir beobachtete Struktur des Ortes, die dort stattfindenden alltäglichen Handlungen und die Erzählungen dieser Handlungen und der Orte aus Sicht der deutschen Finanzmanager.

Mein Aufsatz knüpft an Studien an, die eine interne Homogenität der mobilen Professionals bezweifeln und vielmehr die Relevanz von sozialen 
Identitätsdimensionen wie Ethnizität (Leonard 2010), nationaler Identität (Butcher 2009, Armbruster 2010) oder Geschlecht (Coles/Fechter 2007, Walsh 2011) für die Alltagspraktiken der mobilen hochqualifizierten Migrant_ innen belegt haben. Die „transnational class“ (Sklair 2001) ist heterogen und muss spezifischer betrachtet werden. Daher habe ich in beiden Städten dort arbeitende deutsche Finanzmanager befragt, die sich in verschiedenen sozialen Identitätsdimensionen gleichen: Sie sind Weiß, männlich und sie sind als hoch-qualifizierte Finanzmanager Angehörige der globalen Elite.

Zunächst muss jedoch geklärt werden, welche Kriterien es rechtfertigen von einer sozialen Identitätsdimension globale Elite auszugehen. Denn nur mit einer solchen Klärung wird es im Folgenden möglich ihre konkrete Ausprägung in Abhängigkeit vom Arbeitsort zu betrachten.

\section{Die globale Elite}

Die soziale Identitätsdimension globale Elite lässt sich durch ihren privilegierten Zugang zu Ressourcen und ihre gegenseitige Verknüpfung in sozialen und beruflichen Netzwerken charakterisieren. Ein weiteres Element ist jedoch auch eine diskursive Konstruktion als Elite (Woods 1998). Beispielsweise werden diese, wie Boltanski und Chiapello (2003) darlegen, in der Managementliteratur als ein neuer Typus von Managern entworfen, die in ihren Handlungen und Lebensentwürfen als globale Netzwerkbildner präsentiert werden. Auch wenn, wie Hartmann (2009) zeigt, die Karrieremuster von Topmanagern in den meisten Fällen immer noch traditionellen nationalen Karrieremustern entsprechen, so werden hoch-qualifizierte Manager diskursiv als globale Netzwerkbildner verhandelt. Zu diesem diskursiven Entwurf passend berichten auch meine Interviewpartner beständig davon, wie sie ihre Lebensplanung den Ansprüchen des beruflichen Erfolgs und der notwendigen Netzwerkbildung zurückstellen. Damit unterscheiden sie sich erheblich von einer „Leisure Class“(Veblen 1985), die durch ihren gesicherten Status und Wohlstand von den Mühen der Lohnarbeit entbunden ist. Meine Interviewpartner stellen immer wieder ihren hohen Arbeitseinsatz und ihre Bereitschaft, das Private dem beruflichen Erfolg unterzuordnen, in das Zentrum ihrer Erzählungen und Selbstdefinitionen. Diese Erzählung ist damit verbunden, sich von denen, die dies nicht machen und nicht etwas Neues wagen, da sie weiterhin in Deutschland arbeiten, zu distanzieren. So führt ein Bankmanager in London aus:

„Es gibt viele die nicht gehen wollten. Es gibt viele die nicht äh die sozialen Sicherheiten die es angeblich in Deutschland gibt. Die es gibt ... aufgeben wollten ja und da sind auch einige wie soll ich sagen ... dann jahrelang am Fenster gesessen in Frankfurt so was nennt sich dann Window Seat ja wenn du nichts mehr wichtiges zu tun kriegst, wenn Du Deine Statussymbole da hast und Dein Schreibtisch und so aber eigentlich äh ... viel Zeit hast Zeitung zu lesen und so. Sie werden Dich nicht los und wenn Du mit den Abfindungen die sie Dir anbieten nicht einverstanden bist dann bleibst Du halt da sitzen ja .. und rechnest Deine Rente hoch und und und all diese Kleinkram, ich sag mal wirklich Kleinkramsachen. Äh ... ich bin gegangen, das war 96 und 
dann ist die Familie nachgekommen .. und das war natürlich dann ein äh ... eine sehr große Umstellung hier.“ (Anmerkung: Die Unterstriche kennzeichnen eine besondere Betonung).

Der Schritt an einen Arbeitsplatz im Ausland wird in diesen Erzählungen zu einem mutigen abenteuerlichen Unterfangen. Es ist gerade dieses Selbstverständnis von Mutigkeit und Flexibilität, das in den Erzählungen der Finanzmanager als Distinktionselement gegenüber den aus ihrer Sicht unmutigen und unflexiblen Managern, die in Deutschland bleiben, ausgedrückt wird und ein Kernelement der Aufführung der Identitätsdimension globale Elite darstellt. Wie sich zeigen wird, ist das Selbstverständnis der mobilen Finanzmanager von Mutigkeit und Flexibilität nicht an jedem Arbeitsort gleich ausgeprägt.

\section{Die Performanz als globale Elite in London}

Der nördlich an der Themse gelegene zentrale Stadtteil City of London weist nur eine kleine Fläche von $2,6 \mathrm{~km}^{2}$ auf, in der sich allerdings eine hohe Zahl von Finanzinstitutionen konzentrieren. Die deutschen Finanzmanager in London markieren die City of London als ,Zentrum der Wirtschaft'. Für sie ist die City ein Zentrum mit echter Tradition, oder wie ein Manager in einer Differenzierung der historischen Gebäude in London zu den Gebäuden in anderen Städten sagt: „Hier ist es kein Museum, das ist real“. Ihre Blicke auf die City sind geleitet durch die Wahrnehmung von Gebäuden, die eine symbolische Markierung als traditionelles Zentrum bestätigen. Gebäude, die aber auch durch eine bestimmte Stadtplanung in Szene gesetzt werden und sich in den Blick auf die Skyline aufdrängen sollen, wie zum Beispiel die Kathedrale St. Pauls (Jacobs 1996). In dem traditionellen Zentrum arbeiten zu können, also Eintritt in das „Zentrum der Wirtschaft“ zu haben, wird von den deutschen Finanzmanagern als eine Auszeichnung ihrer persönlichen Leistungsfähigkeit betrachtet, mit der sie sich im Konkurrenzkampf um den Eintritt in das Zentrum gegenüber anderen Finanzmanagern durchgesetzt haben. Denn, so sagt einer:

„Nach London will jeder [...] Das wird schon im Vorfeld genau ausgesucht, passt das Profil, passt die Anforderung.“

Mit dem Aufenthalt der Finanzmanager in der City of London fühlen sie sich selbst als Teil des Zentrums. Hier zu arbeiten, wird von ihnen als Aufwertung der eigenen Person im Konkurrenzkampf gesehen und manifestiert sich als Konkurrenzvorteil im eigenen Lebenslauf. So sagt ein Finanzmanager:

„Zwei Jahre oder ein Jahr London Berufserfahrung, das zahlt sich immer aus im Lebenslauf.“

Das Image der deutschen Finanzbeschäftigten von der City als dem traditionellen Zentrum übersetzt sich aus ihrer Perspektive in besondere Erwartungen, die sie erfüllen müssen, um somit selbst dazu gehören zu können. Daher erscheint eine Erzählung des Arbeitsalltags für die deutschen Finanzmanager nur vollständig, wenn sie auf ihre besonders hohe Arbeitsleistung hinweisen, die sie hier vollbringen müssen. In der City zu sein, ist für sie unmittelbar damit verknüpft, viel und lange zu arbeiten. Das Image vom Zentrum ist für 
die Finanzmanager mit der Erwartung und dem Gefühl verbunden, dass ihr Aufenthalt im Zentrum mit einer notwendigen Anpassung an eine besonders hohe Arbeitsleistung korrespondieren muss. Aus ihrer Perspektive gilt: Wer im Zentrum sein darf, der muss auch eine besonders herausragende Leistung bringen und fühlt sich dort unter einem Anpassungsdruck diesem Image zu entsprechen. Die Erzählung von dieser Anpassung ist so ein ständiges Motiv in den Erzählungen ihres Alltags in der City of London. Wie mächtig diese symbolische Markierung ist und wie stark diese das Empfinden der deutschen Finanzmanager in der City beeinflusst, zeigt sich besonders darin, dass dieses Image von der besonders arbeitsfordernden City nicht der statistischen Realität standhält, aber trotzdem ein dominantes Motiv ihrer Erzählungen ist. So schreibt der „City Economy Digest“, offensichtlich selbst überrascht von dem Ergebnis, über die durchschnittlich erbrachte Arbeitszeit in der City, dass diese mit durchschnittlich 37,2 Stunden die niedrigste in London und in Großbritannien ist (Corporation of London 2003). Auch wenn hieraus keine direkte Aussage über die Arbeitszeit der entsandten deutschen Finanzmanager abzuleiten ist, so ist es doch interessant, dass das Image, das die deutschen Finanzmanager von der City als Ganzes haben, nämlich das des besonders fordernden Zentrums, so stark ist, dass es ihre Empfindung leitet. Sie fühlen sich hier unter einem Anpassungsdruck, selbst diesem Image entsprechend zu handeln und sich als besonders arbeitsam zu präsentieren.

Ihr Gefühl, sich in der City an einem Ort zu befinden, an dem aufgrund dieser Zentrumsfunktion von ihnen eine besondere Arbeitsleistung und Unterordnung unter die Anforderungen der City erwartet wird, korrespondiert mit ihren alltäglich gemachten Erfahrungen und ihren daran angepassten Handlungen von Hektik, wie dem Ignorieren der Fußgängerampeln, den zielstrebigen Bewegungen auf der Straße und dem Verzicht auf ein deutsches Mittagsessen, zugunsten eines schnellen Essens am Arbeitsplatz. Denn, wie einer ausführt, ist

„eine Mittagspause als solche, wie wir sie kennen, eine Stunde mal Abstand nehmen, mal rausgehen oder so, hier im Tagesrhythmus in der City of London für meine Begriffe nicht vorgesehen."

Die deutschen Finanzmanager fühlen sich als Teil einer ausgewählten Gruppe, die es geschafft hat, aufgrund ihrer Leistungsfähigkeit in die City zu passen, und die sich in ihren Handlungen dafür den Anforderungen der City anpassen. Neben der hohen Arbeitsleistung, strengen Kleidungsregeln und der Hektik des Ortes wird von ihnen auch immer wieder eine Notwendigkeit zur sozialen Netzwerkbildung formuliert. Diese Anforderung verdeutlicht sich mit einem Blick auf die zahlreichen Pubs, Cafés und Restaurants, die sich zwischen den Bürogebäuden der City befinden. Ein Interviewpartner macht dies deutlich anhand des Aufforderungscharakters des Tagesrhythmus der City, indem die informelle Netzwerkarbeit einen eigenen Platz hat. Er berichtet:

„75 \% aller Mitarbeiter gehen regelmäßig nach Dienstschluss auf einen Social Drink, das hat Vor- und Nachteile. Der Nachteil ist halt das regelmäßige Trinken, du wirst Alkoholiker. Das ist halt London City.“

Elemente wie das fehlende Lächeln auf der Straße, die fehlende Ablenkung und Irritation des Blicks durch den ,Anderen“, der Mangel an Ruhebuchten 
auf den Straßen der City machen die City für die deutschen Finanzmanager mit dem Überschreiten der Grenzen der City zu einem exklusiven Ort der Konkurrenz. Sie empfinden die City als Ort, an dem aus ihrer Perspektive alles darauf ausgerichtet ist, dem Funktionieren des Finanzwesens zu dienen. Das beschreibt ein Interviewpartner so:

„Es lebt halt von den Banken und den Leuten, die hier halt arbeiten, und es ist halt wirklich nur drauf ausgerichtet, dem zu dienen.“

London wird von den deutschen Finanzmanagern als sozial gespaltene Stadt der Kämpfe und Gefahren betrachtet. Eigene Stadtteile und die Stadtteile der ,Anderen ' werden von Ihnen klar benannt, klassifiziert und mit symbolischen Markierungen versehen. Die Stadtteile der weniger Privilegierten werden für sie als globale Elite als gefährlich markiert und eine Durchquerung vermieden. So führt einer aus:

„Selbst äh der nördliche Teil der Docklands, der high fashionable heute ist, aber da gibt es hohe Lagerhäuser aus der alten Zeit, da möchte ich abends nicht zu Fuß durchgehen.“

Ein anderer erklärt:

„Großstädte bringen immer ein gewisses Chaos mit sich. Unorganisiertheit, auch Dreck, muss man wirklich sagen. Auch gewisse Spannungen in gewissen Gegenden, wo dann also wirklich ein höherer Ausländeranteil ist, äh, Schwarze, Inder vielleicht nicht so sehr, aber Schwarze schon. Wenn Sie nach Brixton zum Beispiel gehen, da sehen Sie schon oftmals, ich will nicht sagen oftmals, aber da kann es schon passieren, dass Sie in der Minderheit sind.“

Die deutschen Finanzmanager schützen sich vor den vermeidlichen Gefahren durch die Aneignung eines spezifischen Wissens der sozialen Zusammensetzung der einzelnen Stadtteile, um so zielgerichtet die Stadtteile der ,Anderen' meiden zu können. Einer erklärt wie er auf Basis eines solchen Erlernens mögliche Wohnorte für ihn identifiziert hat und dabei die Wohnorte der ,Anderen“ ausgeschlossen hat:

„Dann habe ich mir angesehen auf den Straßen was für Autos stehen da und je nachdem wie die Autos sind, wie gepflegt die sind, ist das Mittelklassewagen, höher oder sind das alles alte Schüsseln, können Sie sehr genau sagen was da für Leute wohnen und höchstwahrscheinlich auch welche Leute da durchlaufen. Das ist ein gutes Indiz. Dann äh wie gepflegt sind die Gärten, wie gepflegt sind die Häuser an sich. ... Dann sehen Sie auch schon wie ist die Zusammensetzung von den Leuten. Ich will jetzt nicht sagen wie sind die verschiedenen Herkünfte, sondern vielmehr von dem Bekleidungsstil. Laufen da jetzt viele tagsüber in ihren Jogginghosen rum oder mit dicken Goldketten und sehen eher düster aus. Oder habe ich eben morgens die Leute, die in Krawatte, Anzug äh da aus dem Haus gehen. Dann können sie das in etwa grob abschätzen ähm, ja. Sicherlich irgendwie Erfahrung.“

Die Performanz als globale Elite in London ist für die deutschen Finanzmanager davon bestimmt die eigene hohe Leistungsfähigkeit in der als Zentrum 
symbolisch markierten City darzustellen. Die Markierung als symbolisch markiertes traditionelles Zentrum gründet sich in der Geschichte Londons als koloniales Zentrum, von dem aus die koloniale Peripherie (wie Singapur) beherrscht wurde.

\section{Die Performanz als globale Elite in Singapur}

Am südlichen Ufer des Singapore River schließt der Central Business District (CBD) an, der Teil des historischen Zentrums (Downtown Core) des Stadtstaates ist. Hier konzentrieren sich die Bürogebäude internationaler Finanz- und Dienstleistungsunternehmen. In der Kolonialzeit wurde Singapur zu einem wichtigen Hafen, Umschlagplatz und regionalem Zentrum durch die britische Kolonialmacht entwickelt. Im Downtown Core finden sich um den an den britischen Kolonialherren und Gründer des modernen Singapurs erinnernden Raffles Place neben Hochhäusern auch architektonische Zeugnisse dieser Zeit.

Anders als London wird Singapur von den deutschen Finanzmanagern in seiner historischen Rolle als kolonialer Außenposten betrachtet. Für sie hat daher der CBD im heutigen Singapur längst nicht die zentrale Position in der Weltwirtschaft, die sie der City of London beimessen. Der CBD in Singapur wird von ihnen zwar auch als ein Zentrum gesehen, aber eher als ein untergeordnetes, regionales Zentrum in Asien. Und Asien ist in ihrer Perspektive weit weg vom zentralen Geschehen und vom Hauptsitz des eigenen Finanzunternehmens. Daher sehen sich meine Interviewpartner in Singapur als „hier draußen“. Einer sagt beispielsweise:

„Da ist ein Zeitunterschied [von 7 Stunden], da sind sie schon eine ganze Ecke selbstständiger hier draußen.“

„Draußen“ im CBD von Singapur sehen sich die deutschen Finanzmanager als etwas unabhängiger und „selbstständiger“ als im Zentrum in der City of London. Auch die kolonialen Gebäude betrachten die Finanzmanager anders als in London. Sie werden von ihnen in Singapur eher als Zeugnis einer abhängigen Entwicklung, als Beweis der Rolle als traditionell beherrschter kolonialer Außenposten gesehen. Als Gebäude die nur sie, als die Nachfahren der Erbauer, richtig würdigen können. So sagt ein Finanzmanager:

„Das historische Zentrum von Singapur, da mit City Hall, mit St. Andrews Cathedral, mit Chijmes, das finde ich angenehm. Das ist schön zum Gehen, weil man da die Gebäude sieht im Raum, die Aussagen haben und ein bisschen Geschichte zeigen und nicht nur kaufen, kaufen, kaufen und essen, essen, essen, ja“.

Ihr eigenes Interesse an historischen Gebäuden stellen meine Interviewpartner häufig gerade im Gegensatz zu den Interessen der Singapurer dar. Die deutschen Finanzmanager sehen sich selbst im Unterschied zu den Singapurern als interessiert an Gebäuden, die „Aussagen haben und ein bisschen Geschichte zeigen“. Solche Gebäude sind aus ihrer Perspektive gerade die Gebäude der ehemaligen, weißen, britischen Kolonialherrscher. Ihre eigene Identität als Weiße entsteht dabei mit dem gezeigten Interesse an historischen Gebäuden, die „Aussagen haben“, die Interessen der ,Anderen“ sehen sie dabei als 
gegensätzlich zu den eigenen Interessen. Die deutschen Finanzmanager sehen sich selbst in der Lage, die historischen Gebäude zu würdigen und die „Aussagen“ der Gebäude verstehen zu können. Diese Befähigung, die sich als Identitätsbaustein zum Weißsein verstehen lässt, sehen sie im Gegensatz zu den Fähigkeiten der ,Anderen', den asiatisch stämmigen Singapurern, denen sie diese Fähigkeit nicht zuschreiben, denn so sagt ein Interviewpartner: „kulturell spielen wir sowieso auf anderen Ebenen“.

Die deutschen Finanzmanager betrachten sich selbst in Singapur als diejenigen, die - im Gegensatz zu den asiatisch stämmigen Singapurern - in der Lage sind Kultur zu verstehen und dafür Interesse zu entwickeln. Dass sich dabei das, was sie als Kultur ansehen, gerade auf die historischen Gebäude der weißen britischen Kolonialherren bezieht und die Finanzmanager sich selbst als diejenigen sehen, die diese verstehen und daher daran Interesse entwickeln können, zeigt auf, dass für ihre Produktion einer weißen Identität in Singapur die koloniale Geschichte der Stadt von eminenter Bedeutung ist. Für die deutschen Finanzmanager wird das heutige Singapur in dieser Tradition stehend betrachtet und erlebt. Die Performanz einer weißen Identität erfolgt in Abhängigkeit zu der Geschichte Singapurs als koloniale Stadt (Yeoh 2001); zu einer Stadt, der das ,kulturelle aus Perspektive der weißen Finanzmanager von den weißen Kolonialherren eingeprägt wurde und das heute wie der neu errichtete Konzertsaal „The Esplanade“ zur Befriedigung ihrer speziellen kulturellen Bedürfnisse erstellt wird (zu solchen postkolonialen Kontinuitäten in den Einstellungen und Praktiken der heutigen Expatriates siehe auch Fechter/Walsh 2010).

Anders als in der City of London - in der sich die Finanzmanager, wie gezeigt, an einem Ort der Konkurrenz und der Forderung fühlen - fühlen sie sich im CBD von Singapur an einem Ort, an dem sie mit ihren Eigenschaften als ,Foreign Talents' benötigt werden. Sie erleben sich hier als Mitglied der kleinen Gruppe von westlichen Expatriates, die sie selbst von den ,Anderen“, den vielen hier arbeitenden Dienstleistungsmanagern aus Asien, abgrenzen. Denn diese asiatischen Manager werden von den deutschen Finanzmanagern als unfrei, unkreativ und unflexibel beschrieben und entsprechen damit nicht dem Image, das man von sich selbst als Teil der globalen Elite hat. Der wirtschaftliche Aufschwung Singapurs wird von den deutschen Finanzmanagern daher zu einem bedeutenden Teil auch als Erfolg der Expatriates gesehen, deren angenommene Kreativität erst den wirtschaftlichen Aufschwung des Inselstaates ermöglicht habe. Die deutschen Finanzmanager fühlen sich in Singapur als begehrte Gruppe, für deren Wohlbefinden der Staat Singapur einiges tut. So sagt ein Interviewpartner:

„Die wollen ja im Prinzip eigentlich gerade Foreign Talents, wie das hier heißt, sozusagen anziehen, auch den Platz für diejenigen ein bisschen heimisch machen, in Anführungsstrichen, so dass die Leute sich wohl fühlen und dann auch einfach hier bleiben.“

Die Abhängigkeit des CBD von dem fernen Zentrum spiegelt sich für sie in dem eigenen Aufenthalt wieder: Sie arbeiten hier, da ihre besonderen Fähigkeiten in dieser abhängigen Stadt benötigt werden. Im Unterschied zur City of London wird eine Exklusivität des Ortes von ihnen im CBD so nicht gespürt. Hier im Außenposten sind die,Anderen` allgegenwärtig, nicht nur als asiatisch 
stämmige Finanzmanager, sondern auch als diejenigen, die hier in den vorhandenen Malls einkaufen, in den Hawker Centern (lokale Essenstände) essen oder sich an den Cafés am Singapore River ausruhen. Elemente wie schattige Ruhebuchten, die klimatisierten Gänge der Malls oder ihre Mittagspause in den Hawker Centern lassen den CBD für die Finanzmanager weniger als stressigen Ort der Forderung spürbar werden, sondern mehr als Ort, an dem einiges für das eigene Wohlbefinden geboten wird; nicht nur gegen die Transpiration in den Tropen, sondern auch gegen das mittägliche Hungergefühl sind ihre Körper hier gut geschützt. Ihr Gefühl im CBD gebraucht zu sein korrespondiert mit dem Gefühl sich als globale Elite in Singapur nicht im Konkurrenzkampf mit anderen Finanzmanagern zu befinden.

\section{Intersektionalität der globalen Elite mit anderen privilegierten Identitätsdimensionen und ihre ortsabhängigen Differenzierungen}

Im abschließenden Abschnitt arbeite ich die spezifische Intersektion der Globalen Elite mit der Identitätsdimension des Weißseins und von Männlichkeit heraus. Die deutschen Finanzmanager begegnen der City of London mit dem Image als das exklusive, traditionelle Zentrum und dem Central Business District in Singapur mit dem Image von einer peripheren, kolonialen Stadt. Es sind symbolische Markierungen die nicht nur ihre Begegnungen mit dem Ort prägen, sondern auch die interkulturellen Begegnungen an dem Ort. Beide symbolischen Markierungen sind verwurzelt in den besonderen historischen Entwicklungen der Orte als ,Imperial City und als ,Colonial City' im Weltsystem (King 1990). Diese symbolischen Markierungen übersetzen sich im Kontakt mit dem Ort und seinen Strukturen in eine spezifische Performanz der Finanzmanager.

Es wurde deutlich, dass die genaue Ausprägung der Identitätsdimensionen innerhalbeiner gewissen Bandbreitein Abhängigkeit vom Ortihrer Performanz und Verkündung variiert. Die Identitätsdimension als globale Elite wird von den deutschen Finanzmanagern in Abhängigkeit vom Arbeitsort unterschiedlich prononciert aufgeführt. In der City of London wird diese deutlicher als Eigenschaft präsentiert, mit der sie überhaupt Zutritt in das Zentrum erlangt haben und mit der die eigene Leistungs- und Konkurrenzfähigkeit deutlich wird. Im CBD wird hingegen die Identitätsdimension als globale Elite stärker als Fähigkeit, die Herausforderungen der Fremdheit, also das ,draußen' zu meistern, aufgeführt. Ihre Erzählungen sind weniger davon dominiert von der Bewältigung der starken Arbeitslast zu berichten, sondern mehr davon, über die Bewältigung der Herausforderung, die in der Begegnung mit der Fremdheit liegt, zu berichten. In ihrer Position als globale Elite fühlen sie sich hier unangefochten, denn sie werden aus ihrer Perspektive benötigt und dienen der Fortentwicklung Singapurs. Sie fühlen sich nicht bedroht und haben das Gefühl willkommen zu sein. Als deutscher Finanzmanager in Singapur spüren sie beides, das Ansehen in Deutschland als jemand, der mutig Asien bereist und das Ansehen in Singapur als jemand, der nötig für das Vorankommen des Landes ist.

Das Weißsein in der City of London erscheint den Finanzmanagern als normale Identitätsdimension, deren Eigenschaften wie Vernunft, Kreativität und 
Handlungsfähigkeit von ihnen nicht weiter herausgestellt werden und daher in den Interviews nicht thematisiert wird. In Abgrenzung und in Konkurrenz zu den anderen Weißen ist hier die Aufführung der Identitätsmerkmale der globalen Elite notwendiger, die hier getragene feine Kleidung lässt sich somit auch als Ausdruck dieser sozialen Differenzierungsnotwendigkeit von anderen Weißen verstehen. Im CBD von Singapur hingegen wird das Weißsein von ihnen im Kontrast zu den ,Anderen', den Nicht-Weißen, herausgestellt. Eigenschaften der globalen Elite, wie eine hohe Arbeitsleistung und berufliche Flexibilität sind hier weniger wichtige Differenzierungsmerkmale als die Eigenschaften des Weißseins, die von den Finanzmanagern in Differenz zu den ,Anderen' hervorgehoben werden und den eigenen Aufenthalt in dem Stadtstaat begründen.

Anders als mit einem Umzug nach London übernehmen die deutschen Finanzmanager nach einem Umzug nach Singapur häufiger die Rolle als alleinverdienender Versorger der mitgereisten Familie. Für die mitgereisten Frauen ist es schwierig eine Arbeitserlaubnis zu bekommen, so dass es große Hürden für sie gibt selbst der Lohnarbeit nachzugehen. Stattdessen organisieren sie den Haushalt, die Kindererziehung, die Aufrechterhaltung der sozialen Netzwerke nach Deutschland und den Aufbau neuer sozialer Netzwerke in Singapur (häufig zu anderen Expatriates). Der Umzug nach Singapur geht somit häufig für die Frauen mit einer Aufgabe der eigenen Lohnarbeit und einem Wandel der Performanz von Weiblichkeit einher. Auch die Performanz von Männlichkeit der Finanzmanager ändert sich, da diese nun alleine das Familieneinkommen sichern. Verbunden mit dem Gefühl als Weißer und als globale Elite begehrt zu sein, kommt es auch zu Performanz von Männlichkeit, die heute in Deutschland eher irritieren würden. Dies erläutert ein Interviewpartner am Beispiel der Aufführung von Männlichkeit eines deutschen Freundes

„der hier mit einer Malayin verheiratet ist, der aber eigentlich auch schon sehr asiatisch ist, auch in seinem, sagen wir mal Machogehabe, auch gegenüber Frauen. [...] Wir haben hier mal zusammen Essen gemacht, da gab's Shrimps, da pult er nicht die Shrimps, sondern seine Frau pult sie für ihn. Oder wenn Frauen was sagen zu bestimmten Themen, dann lässt er das unter den Tisch fallen.“

Der Ansatz der Intersektionalität stellt mit der Analyse der Verschränkung verschiedener Dimensionen sozialer Diskriminierung einen innovativen Ansatz zur Erforschung sozialer Ungleichheit dar. Wie mein Aufsatz gezeigt hat, eignet sich dieser Ansatz auch um die häufig unsichtbar bleibenden sozialen Privilegierungen zu analysieren. Ebenso wie verschiedene Diskriminierungsformen sind auch ihr Gegensatz, die Privilegierungsformen, in ihrer Verschränkung besser zu verstehen. Die Performanz der privilegierten Identitäten erfolgt dabei nicht unabhängig vom Ort. Vielmehr wurde in meinem Aufsatz deutlich, dass die spezifische Stadt und ihrer Geschichte die Art der Performanz und die spezifische Intersektion der privilegierten Identitätsdimensionen mit beeinflussen.

Die Publikation dieses Beitrags wurde durch den Open-Access-Fonds der Universitätsbibliothek der Goethe-Universität Frankfurt ermöglicht. 


\section{Autor_innen}

Lars Meier, Soziologe und Geograph, hat die Arbeitsschwerpunkte Soziologie sozialer Ungleichheit, Stadt- und Raumsoziologie, soziale Transformationen und qualitative Methoden. lars.meier@hawk.de

\section{Literaturverzeichnis}

Alderson, Arthur S. / Beckfield, Jason / Sprague-Jones, Jessica (2010): Intercity relations and globalisation. The evolution of the global urban hierarchy. In: Urban Studies 47/9, 1899-1923.

Armbruster, Heidi (2010): Realising the self and developing the african - german immigrants in namibia. In: Journal of Ethnic and Migration Studies 36 (8), 1229-1246.

Bauman, Zygmunt (1998): On glocalisation. Or globalization for some, localisation for other. In: Thesis Eleven 54, 37-49.

Bhabha, Homi (1994): The Location of Culture. London/New York: Routledge.

Boltanski, Luc / Chiapello, Ève (2003): Der neue Geist des Kapitalismus. Konstanz: UVK.

Bourdieu, Pierre (1997): Ortseffekte. In: Pierre Bourdieu (Hg.), Das Elend der Welt. Konstanz: UVK, 117-123.

Bourdieu, Pierre (2005): Was heißt sprechen? Zur Ökonomie des sprachlichen Tausches. Wien: Braumüller.

Brah, Avtar (1996): Cartographies of Diaspora. London: Routledge.

Budde, Jürgen (2013): Das Kategorienproblem. Intersektionalität und Heterogenität. In: Elke Kleinau / Barbara Rendtorff (Hg.), Differenz, Diversität und Heterogenität in erziehungswissenschaftlichen Diskursen. Opladen: Verlag Barbara Budrich, 27-46.

Butcher, Melissa (2009): Ties that bind. The strategic use of transnational relationships in demarcing identity and managing difference. In: Journal of Ethnic and Migration Studies 35/8, 1353-1371.

Butler, Judith (1991): Das Unbehagen der Geschlechter. Frankfurt/Main: Suhrkamp.

Carroll, William K. / Fennema, Meindert (2002): Is there a transnational business community? In: International Sociology 17/3, 393-419.

Castells, Manuel (2000): The Rise of the Network Society. Oxford: Blackwell.

Charmaz, Kathy (2014): Constructing grounded theory. London: Sage.

Çınar, Alev / Bender, Thomas (Hg.) (2007): Urban Imaginaries - Locating the Modern City. Minneapolis: University of Minnesota Press.

Coles, Anne / Fechter, Anne-Meike (Hg.) (2007): Gender and family among transnational professionals. London: Routledge.

Collins, Patricia Hill (1998): It's all in the family: intersections of gender, race, and nation. In: Hypatia 13/3, 62-82.

Corporation of London (2003): City Economy Digest No 4. London.

Crenshaw, Kimberlé W. (1989): Demarginalizing the intersection of race and sex. A black feminist critique of antidiscrimination doctrine, feminist theory and antiracist politics. In: The University of Chicago Legal Forum 1/1989, 139-167.

Cresswell, Tim (1996): In Place/Out of Place. Minneapolis: University of Minnesota Press.

Fechter, Anne-Meike / Walsh, Katie (2010): Examining 'expatriate' continuities - postcolonial approaches to mobile professionals. In: Journal of Ethnic and Migration Studies 36 (8), 1197-1210.

Fechter, Anne-Meike (2007): Transnational Lives - Expatriates in Indonesia. London: Routledge.

Flick, Uwe (2008): Triangulation - Eine Einführung. Wiesbaden: VS Verlag.

Frank, Susanne (2010): Gentrifizierung und Suburbanisierung im Fokus der Urban Gender Studies. In: Sybille Bauriedl / Michaela Schier / Anke Strüver (Hg.), Geschlechterverhältnisse, Raumstrukturen, Ortsbeziehungen. Münster: Westfälisches Dampfboot, 26-47.

Frankenberg, Ruth (1993): White Women Race Matters - The Social Construction of Whiteness. Minneapolis: University of Minneapolis Press.

Friedmann, John (1995): Where we stand - a decade of world city research. In: Paul L. Knox / Peter J. Taylor (Hg.), World Cities in a World System. Cambridge: Cambridge University Press, 21-47. 
Hall, Stuart (1996): Who needs 'identity'? In: Stuart Hall / Paul du Gay (Hg.), Cultural Identity. London: Sage, 1-17.

Hartmann, Michael (2009): Die transnationale Klasse - Mythos oder Realität? In: Soziale Welt 6o/3, 285-303.

hooks, bell (1981): Ain't I a Woman?: Black Women and Feminism. Boston: South End Press.

Jackson, Peter (1989): Maps of Meaning - An introduction to cultural geography. London: Unwin Hyman.

Jacobs, Jane M. (1996): Edge of Empire - Postcolonialism and the city. London/New York: Routledge.

Kerner, Ina (2013): Critical Whiteness Studies: Potentiale und Grenzen eines wissenspolitischen Projekts. In: Feministische Studien. 31/2, 278-293.

King, Anthony (1990): Urbanism, Colonialism, and the World Economy. London/New York: Routledge.

Knorr-Cetina, Karin / Bruegger, Urs (2002): Global microstructures - the virtual societies of financial markets. In: American Journal of Sociology 107/4, 905-950.

Korsnes, Olav / Heilbron, Johan / Hjellbrekke, Johs / Bühlmann, Felix / Savage, Mike (Hg.) (2017): New Directions in Elite Studies. London: Routledge.

Leonard, Pauline (2010): Work, identity and change? Post/colonial encounters in Hong Kong. In: Journal of Ethnic and Migration Studies 36/8, 1247-1263.

Ley, David (2004): Transnational spaces and everyday lives. In: Transactions of the Institute of British Geographers 29/2, 151-164.

Lutz, Helma / Herrera Vivar, Maria Teresa / Supik, Linda (Hg.) (2010): Fokus Intersektionalität. Bewegungen und Verortungen eines vielschichtigen Konzeptes. Wiesbaden: VS Verlag.

McDowell, Linda (1997): Capital Culture - Gender at work in the city. Oxford/Malden: Blackwell.

Meier, Lars (2007): Working in the Skyline - Images and Everyday Action. In: Frers, Lars / Meier, Lars (Hg.), Encountering Urban Places - Visual and Material Performances in the City. New York/London: Routledge, 119-134.

Meier, Lars (2009): Das Einpassen in den Ort - Der Alltag deutscher Finanzmanager in London und Singapur. Bielefeld: transcript.

Meier, Lars (Hg.) (2015a): Migrant Professionals in the City - Local Encounters, Identities, and Inequalities. New York/London: Routledge.

Meier, Lars (2015b): Learning the city by experiences and images. German finance managers encounters in London and Singapore. In: Meier, Lars (Hg.), Migrant Professionals in the City - Local Encounters, Identities, and Inequalities. New York/London: Routledge, 59-74.

Meier, Lars (2016): Dwelling in different localities - identity performances of a white transnational professional elite in the City of London and the Central Business District of Singapore. In: Cultural Studies 30/3, 483-505.

Ohmae, Kenichi (1990): The Borderless World - Power and Strategy in the Interlinked Economy. New York: Harper Business.

Salt, John (1997): International movements of the highly skilled. In: International Migration Unit Occasional Papers, No. 3, Paris: OECD.

Sassen, Saskia (2001): The Global City. New York, London, Tokio; revised. Princeton: Princeton University Press.

Scambor, Elli / Zimmer, Fränk (Hg.) (2012): Die intersektionelle Stadt. Geschlechterforschung und Medienkunst an den Achsen der Ungleichheit. Bielefeld: Transcript.

Sicotte, Diane (2014): Diversity and intersectionality among environmentally burdened communities in the Philadelphia Metropolitan Area, USA. In: Urban Studies 51/9, 1850-1870.

Sklair, Leslie (2001): The Transnational Capitalist Class. Oxford: Blackwell.

Spradley, James P. (1979): The Ethnographic Interview. New York: Rinehart \& Winston.

Taylor, Peter J. (2004): World City Network - A Global Urban Analysis. London: Routledge.

Valentine, Gill (2007): Theorizing and researching intersectionality: A challenge for feminist geography. In: The Professional Geographer 59/1, 10-21.

Valentine, Gill (2008): Living with difference: reflections on geographies of encounter. In: Progress in Human Geography 32/3, 323-337.

Van Der Pijl, Kees (1998): Transnational Classes and International Relations. London: Routledge. 
Veblen, Thorstein (1985 [1899]): The Theory of the Leisure Class. London: Allen and Unwin.

Yeoh, Brenda S.A. (2001): Postcolonial cities. In: Progress in Human Geography 25/3, 456-468.

Wacquant, Loïc (2017): Mit Bourdieu in die Stadt. Relevanz, Prinzipien, Anwendungen. In: sub \urban. Zeitschrift für kritische Stadtforschung 5/(1/2), 173-196. http://www. zeitschrift-suburban.de/sys/index.php/suburban/article/view/280 (letzter Zugriff am 20.9.2018).

Walby, Sylvia / Armstrong, Jo / Strid, Sofia (2012): Intersectionality: Multiple inequalities in social theory. In: Sociology 46/2, 224-240.

Walgenbach, Katharina(2012): Intersektionalität-eineEinführung.www.portal-intersektionalität.de (letzter Zugriff am 12.6.2018).

Walsh, Katie (2011): Migrant masculinities and domestic space: British home-making practices in Dubai. In: Transactions of the Institute of British Geographers 36/4, 516-529.

Willis, Katie / Yeoh, Brenda / Fakhri, S.M.A.K. (2002): Introduction - transnational elites. In: Geoforum 33, 505-507.

Winker, Gabriele / Degele, Nina (2009): Intersektionalität. Zur Analyse sozialer Ungleichheiten. Bielefeld: Transcript.

Wolfe, Tom (1987): Bonfire of the Vanities. New York: Picador.

Woods, Michael (1998): Rethinking elites - networks, space, and local politics. In: Environment and Planning A 30, 2101-2119.

\section{Intersectionality of Privilege. Performances of a white, mascu- line and global elite}

This article examines the intersectionality of privilege identity dimensions. On the example of German finance managers, the identity performances of a white, masculine and global elite are analysed. This is done with respect to the specific city where the identities are performed. 
